\title{
How new strategies can improve productivity - rMMS microseeding for crystallization and DLS for cryoEM
}

\author{
Patrick Shaw Stewart, Douglas Instruments Ltd, Hungerford, UK (carolyn@douglas.co.uk)
}

Abstract

Random Microseed Matrix-Screening (rMMS), where seed crystals are added automatically to random crystallization screens, is a significant recent breakthrough in protein crystallization [1]. During the ten years since the method was published, understanding of the theoretical advantages of the method has increased [2 - 4], and several practical variations on the basic method have emerged. Important variations that will be discussed include combining seeds from several hits [5], the best methods of selecting hits to optimize [2], and cross-seeding targets with crystals of homologous proteins [6].

Single-particle cryoEM clearly has great potential to determine the structures of macromolecules when crystallographic approaches are not available. The throughput of cryoEM is, however, low, with only one or a few samples being analysed per day. This creates a need for a pre-screening approach to investigate the behaviour of macromolecules in solution. Douglas Instruments has worked closely with Xtal Concepts to develop a screening approach were e.g. 96 wells can be analysed by DLS using only a few microlitres of sample $[8,9]$. This concept will be outlined briefly, with examples.

[1] D'Arcy, Allan, Frederic Villard, and May Marsh. "An automated microseed matrix-screening method for protein crystallization." Acta Crystallographica Section D: Biological Crystallography 63.4 (2007): 550-554.

[2] Shaw Stewart, Patrick D., et al. "Random microseeding: a theoretical and practical exploration of seed stability and seeding techniques for successful protein crystallization." Crystal Growth \& Design 11.8 (2011): 3432-3441.

[3] D'Arcy, A., Bergfors, T., Cowan-Jacob, S. W., \& Marsh, M. (2014). Microseed matrix screening for optimization in protein crystallization: what have we learned?. Acta Crystallographica Section F: Structural Biology Communications, 70(9), 1117-1126.

[4] Shaw Stewart, P., \& Mueller-Dieckmann, J. (2014). Automation in biological crystallization. Acta Crystallographica Section F: Structural Biology Communications, 70(6), 686-696.

[5] Obmolova, G., Malia, T. J., Teplyakov, A., Sweet, R. W., \& Gilliland, G. L. (2014). Protein crystallization with microseed matrix screening: application to human germline antibody Fabs. Structural Biology and Crystallization Communications, 70(8).

[6] Abuhammad, Areej, et al. "Structure of arylamine N-acetyltransferase from Mycobacterium tuberculosis determined by cross-seeding with the homologous protein from M. marinum: triumph over adversity." Acta Crystallographica Section D: Biological Crystallography 69.8 (2013): 1433-1446.

[7] Kolek, S. A., Bräuning, B., \& Shaw Stewart, P. D. (2016). A novel microseeding method for the crystallization of membrane proteins in lipidic cubic phase. Acta Crystallographica Section F: Structural Biology Communications, 72(4), 307-312.

[8] Falke, S., Dierks, K., Blanchet, C., Graewert, M., Cipriani, F., Meijers, R., Svergun, D. and Betzel, C., 2018. Multi-channel in situ dynamic light scattering instrumentation enhancing biological smallangle X-ray scattering experiments at the PETRA III beamline P12. Journal of synchrotron radiation, 25(2).

[9] https://www.douglas.co.uk/cryoem.htm 